\title{
Rhodium-Catalyzed Hydrothiolation of 1,3-Dienes
}

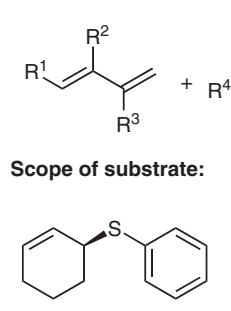

$95 \%$ yield, $r$ r $>20: 1$ er $=99: 1$ (L2)<smiles>C1=CC(SCCc2ccccc2)CCC1</smiles>
$91 \%$ yield, $r r>20: 1$ er $=97: 3(\mathbf{L 2})$

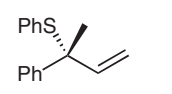

$73 \%$ yield, $r r=12: 1$ er $=96: 4$ (JosiPhos)

Proposed mechanism:

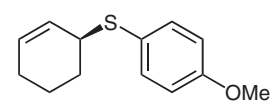

$96 \%$ yield, $r r>20: 1$ er $=98: 2($ L2)<smiles>O=C(O)CCSC1C=CCCC1</smiles>
$79 \%$ yield, $r r>20: 1$ er $=99: 1$ (L2)
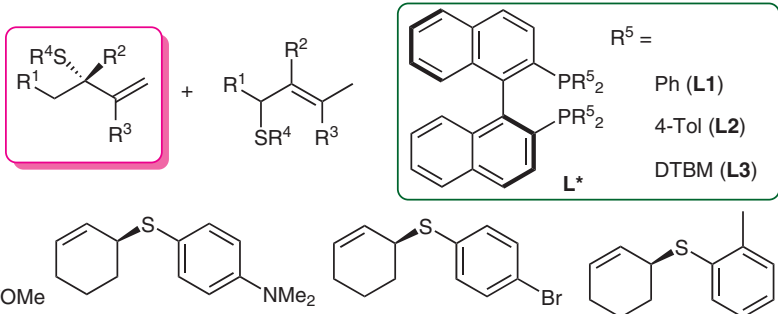

$67 \%$ yield, $r r>20: 1$ er $=98: 2$ (L2)
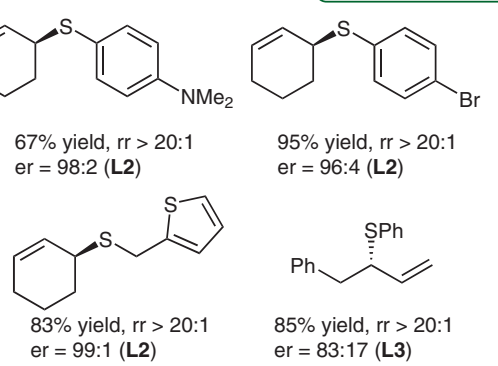

$95 \%$ yield, $r r>20: 1$ er $=96: 4$ (L2)

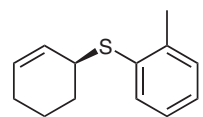

$93 \%$ yield, $r r>20: 1$ er $=99: 1$ (L2)

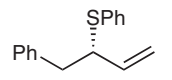

$85 \%$ yield, $r r>20: 1$ er $=83: 17$ (L3)

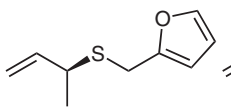

$84 \%$ yield, $r r>20:$ er $=94: 6$

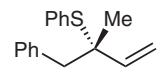

$78 \%$ yield, $\mathrm{rr}>20: 1$ er $=71: 29$ (L3)

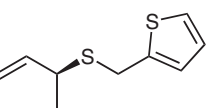

thiols

dienes

sulfides

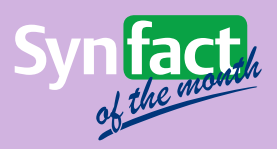

Significance: The development of reactions for the construction of $\mathrm{C}-\mathrm{S}$ bonds is important because molecules essential to life contain this linkage. The addition of thiols to alkenes is a direct and atom-economical method for the formation of $\mathrm{C}-\mathrm{S}$ bonds. The authors have developed an enantioselective addition of thiols to 1,3-dienes catalyzed by a rhodium-chiral bisphosphine ligand complex to give chiral secondary or tertiary allylic sulfides in good to high enantioselectivities.
Comment: The enantioselective hydrothiolation proceeds selectively at the more-substituted double bond. A broad range of functional groups are tolerated in this reaction, and the catalyst loading can be lowered to $0.1 \mathrm{~mol} \%$.

SYNFACTS Contributors: Hisashi Yamamoto, Takahiro Sawano

Synfacts 2018, 14(11), 1145 Published online: 18.10.2018 Dol: 10.1055/s-0037-1611240; Reg-No.: H12417SF 